\title{
Aggregation Operators and the Lipschitzian Condition
}

\author{
J. Jacas and J. Recasens
}

\begin{abstract}
Lipschitzian and kernel aggregation operators with respect to the natural $T$-indistinguishability operator $E_{T}$ and their powers are studied. $A$ t-norm $T$ is proved to be $E_{T^{-}}$ Lipschitzian, and is interpreted as a fuzzy point and a fuzzy map as well. Given an Archimedean t-norm $T$ with additive generator $t$, the quasi-arithmetic mean generated by $t$ is proved to be the most stable aggregation operator with respect to $T$.
\end{abstract}

\section{INTRODUCTION}

Lipschitzian conditions are fulfilled by many maps and operators in fuzzy reasoning. They give stability to the system since the similarity or distance between the outputs is bounded by the corresponding one between the inputs. The Lipschitzian condition appears for example in the study of fuzzy maps [12], vague algebras [6], fuzzy modifiers and fuzzy logic in the narrow sense [16], fuzzy topology [8], extensionality [12] among others and therefore it deserves a deep study.

Lipschitzian aggregation operators have been studied in [4] [5] [13] [14] considering the usual metric on the unit interval. This paper studies the Lipschitzian condition of aggregation operators in a broader sense, i.e. with respect to the natural indistinguishability operator $E_{T}$ and their powers $E_{T}^{p}$ (see definitions below) so that an aggregation operator $h$ is $E_{T}^{P}$ Lipschitzian when

$$
\begin{gathered}
T\left(E_{T}^{p}\left(x_{1}, y_{1}\right), \ldots, E_{T}^{p}\left(x_{n}, y_{n}\right)\right) \leq \\
E_{T}\left(h\left(x_{1}, x_{2}, \ldots, x_{n}\right), h\left(y_{1}, y_{2}, \ldots, y_{n}\right)\right)
\end{gathered}
$$

for all $x_{1}, x_{2}, \ldots, x_{n}, y_{1}, y_{2}, \ldots, y_{n} \in[0,1]$. The meaning is that from similar values we obtain a similar aggregation. The use of $E_{T}$ and $E_{T}^{p}$ assumes the choice of a specific t-norm $T$ and therefore the selection of a particular family of logics where the semantics of the conjunction and the biimplication are given by $T$ and $E_{T}$.

As it will be seen in the paper, when $T$ is the Lukasiewicz t-norm, the $E_{T}$-Lipschitzian condition coincides with the 1Lipschitzian condition with the usual metric on $[0,1]$; not surprisingly, due to the relation between $E_{T}$ and the usual metric on $[0,1]$ in this case.

Easy to state, but interesting, the $E_{T}^{p}$-Lipschitzian condition is equivalent to the extensionality of the aggregation operator (Proposition 3.10).

\footnotetext{
J. Jacas is with the Seccio Matemàtiques i Informàtica, ETS Arquitectura de Barcelona, Universitat Politècnica de Catalunya, Diagonal 649, 08028 Barcelona, Spain (phone: +34 9340163 66; fax: +34 9340163 72; email: joan.jacas@upc.edu).

J. Recasens is with the Secció Matemàtiques i Informàtica, ETS Arquitectura del Vallès, Universitat Politècnica de Catalunya, Pere Serra 1-15, 08190 Sant Cugat del Vallès, Spain (phone: +34 9340178 68; fax: +34 93 40178 49; email: j.recasens@upc.edu).
}

Among other results, it will be proved that if $T$ is a continuous Archimedean t-norm with additive generator $t$ and $h_{t}$ the quasi-arithmetic mean generated by $t$ $\left(h_{t}\left(x_{1}, x_{2}, \ldots, x_{n}\right)=t^{-1}\left(\frac{t\left(x_{1}\right)+t\left(x_{2}\right)+\ldots, t\left(x_{n}\right)}{n}\right)\right)$, then $h_{t}$ is the most stable aggregation operator with respect to $T$ (Proposition 3.22).

Also the t-norm $T$ is not only Lipschitzian with respect to $E_{T}$, but it can be seen as a fuzzy point and a fuzzy map as well (Proposition 3.24, Proposition 3.26) and an aggregation operator $h$ is greater or equal to $T$ if and only if $h$ is $1-E_{T^{-}}$ Lipschitzian.

If in the definition of $E_{T}$-Lipschitzianity we replace the t-norm $T$ by the minimum

$$
\begin{gathered}
\left(\operatorname{Min}\left(E_{T}^{p}\left(x_{1}, y_{1}\right), \ldots, E_{T}^{p}\left(x_{n}, y_{n}\right)\right) \leq\right. \\
\left.E_{T}\left(h\left(x_{1}, x_{2}, \ldots, x_{n}\right), h\left(y_{1}, y_{2}, \ldots, y_{n}\right)\right)\right)
\end{gathered}
$$

, we obtain a generalization of the kernel aggregation operators studied in [17] [13]. Again, if $T$ is the Lukasiewicz t-norm this definition is equivalent to the one given in the above mentioned references.

\section{PReLiminaries}

This section contains some results about t-norms and indistinguishability operators that will be needed later on in this paper. Besides well known definitions and theorems, the power $T^{n}$ of a t-norm is generalized to irrational exponents in Definition 2.4. and it is given explicitly for continuous Archimedean t-norms in Proposition 2.5.

Though many results remain valid for arbitrary t-norms and especially for left continuous ones, for the sake of simplicity, we will assume continuity for the t-norms throughout the paper.

Definition 2.1: A continuous t-norm is a continuous map $T:[0,1] \times[0,1] \rightarrow[0,1]$ satisfying for all $x, y, z, x^{\prime}, y^{\prime} \in$ $[0,1]$

1) $T(x, T(y, z))=T(T(x, y), z)$ (Associativity)

2) $T(x, y)=T(y, x)$ (Commutativity)

3) If $x \leq x^{\prime}$ and $y \leq y^{\prime}$, then $T(x, y) \leq T\left(x^{\prime}, y^{\prime}\right)$ (Monotonicity)

4) $T(1, x)=x$

Since a t-norm $T$ is associative, we can extend it to an $n$-ary operation in the standard way:

$$
\begin{aligned}
& T(x)=x \\
& T\left(x_{1}, x_{2}, \ldots x_{n}\right)=T\left(x_{1}, T\left(x_{2}, \ldots, x_{n}\right)\right) .
\end{aligned}
$$


In particular, $T(\overbrace{x, x, \ldots x}^{n \text { times }})$ will be denoted by $x_{T}^{(n)}$.

If $T$ is continuous, the $n$-th root $x_{T}^{\left(\frac{1}{n}\right)}$ of $x$ with respect to $T$ is defined by

$$
x_{T}^{\left(\frac{1}{n}\right)}=\sup \left\{z \in[0,1] \mid z_{T}^{(n)} \leq x\right\}
$$

and for $m, n \in \mathbb{N}, x_{T}^{\left(\frac{m}{n}\right)}=\left(x_{T}^{\left(\frac{1}{n}\right)}\right)_{T}^{(m)}$.

Lemma 2.2: [18] If $k, m, n \in \mathbb{N}, k, n \neq 0$ then $x_{T}^{\left(\frac{k m}{k n}\right)}=$ $x_{T}^{\left(\frac{m}{n}\right)}$.

Lemma 2.3: Let $x_{1}, \ldots, x_{n} \in(0,1]$ and $n \in \mathbb{N}$. $T\left(x_{1_{T}}^{\left(\frac{1}{n}\right)}, \ldots, x_{n_{T}}^{\left(\frac{1}{n}\right)}\right) \neq 0$.

Assuming continuity for the t-norm $T$, the powers $x_{T}^{\left(\frac{m}{n}\right)}$ can be extended to irrational exponents in a straightforward way.

Definition 2.4: If $r \in \mathbb{R}^{+}$is a positive real number, let $\left\{a_{n}\right\}_{n \in \mathbb{N}}$ be a sequence of rational numbers with $\lim _{n \rightarrow \infty} a_{n}=r$. For any $x \in[0,1]$, the power $x_{T}^{(r)}$ is

$$
x_{T}^{(r)}=\lim _{n \rightarrow \infty} x_{T}^{\left(a_{n}\right)} .
$$

Continuity assures the existence of the limit and its independence from the sequence $\left\{a_{n}\right\}_{n \in \mathbb{N}}$.

Proposition 2.5: Let $T$ be an Archimedean t-norm with additive generator $t, x \in[0,1]$ and $r \in \mathbb{R}^{+}$. Then

$$
x_{T}^{(r)}=t^{[-1]}(r t(x)) .
$$

Proof: Due to the continuity of $t$, we need to prove it only for rational values of $r$.

If $r$ is a natural number $m$, then trivially $x_{T}^{(m)}=$ $t^{[-1]}(\operatorname{mt}(x))$.

If $r=\frac{1}{n}$ with $n \in \mathbb{N}$, then $x_{T}^{\left(\frac{1}{n}\right)}=z$ with $z_{T}^{(n)}=x$ or $t^{[-1]}(n t(z))=x$ and $x_{T}^{\left(\frac{1}{n}\right)}=t^{[-1]}\left(\frac{t(x)}{n}\right)$.

For a rational number $\frac{m}{n}$,

$$
\begin{gathered}
x_{T}^{\left(\frac{m}{n}\right)}=\left(x_{T}^{\left(\frac{1}{n}\right)}\right)_{T}^{(m)}=t^{[-1]}\left(m t\left(x_{T}^{\left(\frac{1}{n}\right)}\right)\right)= \\
t^{[-1]}\left(m t\left(t^{[-1]}\left(\frac{t(x)}{n}\right)\right)\right)=t^{[-1]}\left(\frac{m}{n} t(x)\right) .
\end{gathered}
$$

Let $E(T)=\left\{x \in[0,1] \mid x_{T}^{(2)}=x\right\}$ be the set of idempotent elements of $T$ and $N I L(T)=\left\{x \in[0,1] \mid x_{T}^{(n)}=\right.$ 0 for some $n \in \mathbb{N}\}$ the set of nilpotent elements of $T$.

Definition 2.6: A continuous t-norm $T$ is Archimedean if and only if $E(T)=\{0,1\}$. $T$ is called non-strict or nilpotent when $N I L(T)=[0,1)$ Otherwise it is called strict and $N I L(T)=\{0\}$.

Theorem 2.7: Ling [15] A continuous t-norm $T$ is Archimedean if and only if there exists a continuous decreasing map $t:[0,1] \rightarrow[0, \infty]$ with $t(1)=0$ such that

$$
T(x, y)=t^{[-1]}(t(x)+t(y))
$$

where $t^{[-1]}$ stands for the pseudo-inverse of $t$ defined by

$$
t^{[-1]}(x)= \begin{cases}1 & \text { if } x<0 \\ t^{-1}(x) & \text { if } x \in[0, t(0)] \\ 0 & \text { otherwise. }\end{cases}
$$

$T$ is strict if $t(0)=\infty$ and non-strict otherwise.

$t$ is called an additive generator of $T$ and two additive generators of the same t-norm differ only by a multiplicative constant.

Definition 2.8: The residuation $\vec{T}$ of a t-norm $T$ is defined by

$$
\vec{T}(x \mid y)=\sup \{\alpha \in[0,1] \mid T(x, \alpha) \leq y) .
$$

Definition 2.9: The natural $T$-indistinguishability $E_{T}$ associated to a given t-norm $T$ is the fuzzy relation on $[0,1]$ defined by

$$
E_{T}(x, y)=T(\vec{T}(x \mid y), \vec{T}(y \mid x))=\operatorname{Min}(\vec{T}(x \mid y), \vec{T}(y \mid x)) .
$$

Example 2.10:

1) If $T$ is an Archimedean t-norm with additive generator $t$, then $E_{T}(x, y)=t^{-1}(|t(x)-t(y)|)$ for all $x, y \in$ $[0,1]$.

2) If $T$ is the Lukasiewicz t-norm, then $E_{T}(x, y)=1-$ $|x-y|$ for all $x, y \in[0,1]$.

3) If $T$ is the Product t-norm, then $E_{T}(x, y)=$ $\operatorname{Min}\left(\frac{x}{y}, \frac{y}{x}\right)$ for all $x, y \in[0,1]$ where $\frac{z}{0}=1$.

4) If $T$ is the Minimum t-norm, then $E_{T}(x, y)=$ $\begin{cases}\operatorname{Min}(x, y) & \text { if } x \neq y \\ 1 & \text { otherwise. }\end{cases}$

$E_{T}$ is indeed a special kind of (one-dimensional) $T$ indistinguishability operator (Definition 2.11) [3] and in a logical context where $T$ plays the role of the conjunction, $E_{T}$ is interpreted as the bi-implication associated to $T$ [7].

The general definition of $T$-indistinguishability operator $[22][21]$ is

Definition 2.11: Given a t-norm $T$, a $T$ indistinguishability operator $E$ on a set $X$ is a fuzzy relation $E: X \times X \rightarrow[0,1]$ satisfying for all $x, y, z \in X$

1) $E(x, x)=1$ (Reflexivity)

2) $E(x, y)=E(y, x)$ (Symmetry)

3) $T(E(x, y), E(y, z)) \leq E(x, z)$ (T-transitivity).

Proposition 2.12: [21] Let $\mu$ be a fuzzy subset of $X$ and $T$ a continuous t-norm. The fuzzy relation $E_{\mu}$ on $X$ defined for all $x, y \in X$ by

$$
E_{\mu}(x, y)=E_{T}(\mu(x), \mu(y))
$$

is a $T$-indistinguishability operator on $X$. 
Definition 2.13: Let $E$ be a $T$-indistinguishability operator on a set $X$. A fuzzy subset $\mu$ of $X$ is extensional with respect to $E$ if and only if for all $x, y \in X$

$$
T(E(x, y), \mu(y)) \leq \mu(x) .
$$

Proposition 2.14: Let $E$ be a $T$-indistinguishability operator on a set $X$. A fuzzy subset $\mu$ of $X$ is extensional with respect to $E$ if and only if for all $x, y \in X$

$$
E(x, y) \leq E_{T}(\mu(x), \mu(y)) .
$$

Finally, let us recall in this preliminary section the definition of aggregation operator.

Definition 2.15: [4] An aggregation operator is a map $h$ : $\cup_{n \in \mathbb{N}}[0,1]^{n} \rightarrow[0,1]$ satisfying

1) $h(0, \ldots, 0)=0$ and $h(1, \ldots, 1)=1$

2) $h(x)=x \forall x \in[0,1]$

3) $h\left(x_{1}, \ldots, x_{n}\right) \leq h\left(y_{1}, \ldots, y_{n}\right)$

$$
\text { if } x_{1} \leq y_{1}, \ldots, x_{n} \leq y_{n} \text { (monotonicity). }
$$

The restriction of $h$ to $[0,1]^{n}$ will be denoted by $h_{(n)}$ so that a global aggregation operator $h$ can be split into the family of $n$-ary operators $\left(h_{(n)}\right)_{n \in \mathbb{N}}$.

\section{III. $E_{T}$-LIPSCHITZIAN AND $E_{T}$-KERNEL AGGREGATION OPERATORS}

Lipschitzian and kernel aggregation operators with respect to the natural $T$-indistinguishability operator $E_{T}$ and their powers are a special kind of aggregation operators that generalize the definitions of [13], [17]. Their interest lies in the fact that they are stable operators in the sense that the similarity between the aggregation of two $n$-tuples is bounded by the similarity between them.

It is interesting to point out that the Lipschitzian and kernel conditions are equivalent to extensionality (Proposition 3.10, Proposition 3.28).

Among other results, it will be proved that a t-norm $T$ is $E_{T}$-Lipschitzian and moreover the maps $T_{(n)}$ can be interpreted as fuzzy points of $[0,1]^{n}$ and a fuzzy maps from $[0,1]^{k}$ to $[0,1]^{n-k}$.

Also quasi-arithmetic means are proved to be the more stable aggregation operators.

Proposition 3.1: Let $E$ be a $T$ indistinguishability operator on a set $X$. The fuzzy relation $E^{n}$ defined by

$$
E^{n}(x, y)=T(\overbrace{E(x, y), \ldots, E(x, y)}^{n \text { times }}) \forall x, y \in X
$$

is a $T$-indistinguishability operator.

Corollary 3.2: [20] Let $E_{T}$ be the natural $T$ indistinguishability operator on $[0,1]$ associated to $T$. $E_{T}^{n}$ is a $T$-indistinguishability operator.

The powers $E_{T}^{n}$ of the natural $T$-indistinguishability operators have been studied in relation with antonymy and fuzzy partitions in [20].
Proposition 3.3: Let $E$ be a $T$-indistinguishability operator on a set $X . E^{\frac{1}{n}}$ is a $T$-indistinguishability operator on $X$.

Proof: Reflexivity and symmetry are trivial.

Transitivity: If $E^{\frac{1}{n}}=F$, then $F^{n}=E$. Since $E$ is a $T$-indistinguishability operator, $\forall x, y, z \in X$

$$
\begin{gathered}
F^{n}(x, z) \leq T\left(F^{n}(x, y), F^{n}(y, z)\right)=(T(F(x, y), F(y, z)))_{T}^{(n)} \\
\left(F^{n}(x, z)\right)_{T}^{\frac{1}{n}} \leq\left((T(F(x, y), F(y, z)))_{T}^{(n)}\right)_{T}^{\frac{1}{n}}
\end{gathered}
$$

and from Lemma 2.2

$$
F(x, z) \leq T(F(x, y), F(y, z)) .
$$

Corollary 3.4: Let $E_{T}$ be the natural $T$ indistinguishability operator on $[0,1]$ associated to $T$. $E_{T}^{\frac{1}{n}}$ is a $T$-indistinguishability operator.

Corollary 3.5: Let $E$ be a $T$-indistinguishability operator on a set $X . E^{\frac{m}{n}}$ is a $T$-indistinguishability operator on $X$.

Proof: Propositions 3.1. and 3.3 .

Corollary 3.6: Let $E_{T}$ be the natural $T$ indistinguishability operator on $[0,1]$ associated to $T$. $E_{T}^{\frac{m}{m}}$ is a $T$-indistinguishability operator.

Continuity of the t-norm $T$ allows us to extend the powers of a $T$-indistinguishability operator to positive irrational numbers in the same way as in Definition 2.4.

\section{Example 3.7:}

1) If $T$ is continuous Archimedean with additive generator $t$, then $E_{T}^{p}(x, y)=t^{[-1]}(p|t(x)-t(y)|)$ for all $x, y \in$ $[0,1]$.

2) If $T$ is the Lukasiewicz t-norm, then $E_{T}^{p}(x, y)=$ $\operatorname{Max}(0,1-p|x-y|)$ for all $x, y \in[0,1]$.

3) If $T$ is the Product t-norm, then $E_{T}^{p}(x, y)=$ $\left(\operatorname{Min}\left(\frac{x}{y}, \frac{y}{x}\right)\right)^{p}$ for all $x, y \in[0,1]$ where $\frac{z}{0}=1$.

4) If $T$ is the Minimum t-norm, then $E_{T}^{p}(x, y)=$ $E_{T}(x, y)$ for all $x, y \in[0,1]$.

With the previous results we can relax or strengthen the equivalence relations. Indeed, $E_{T}^{p} \leq E_{T}^{q}$ if and only if $p \geq q$.

Definition 3.8: Let $E$ be a $T$-indistinguishability operator on $[0,1]$. An aggretation operator $h$ is $E$-lipschitzian if and only if $\forall n \in \mathbb{N}, \forall x_{1}, \ldots, x_{n}, y_{1}, \ldots, y_{n} \in[0,1]$

$T\left(E\left(x_{1}, y_{1}\right), \ldots, E\left(x_{n}, y_{n}\right)\right) \leq E_{T}\left(h\left(x_{1}, \ldots, x_{n}\right), h\left(y_{1}, \ldots, y_{n}\right)\right)$.

Let us recall that if we have several $T$-indistinguishability operators $E_{1}, \ldots, E_{n}$ defined on different universes $X_{1}, \ldots, X_{n}$, there are several ways to define a $T$ indistinguishability operator on $X_{1} \times \ldots \times X_{n}$. 
Proposition 3.9: Let $E_{1}, \ldots, E_{n}$ be $T$-indistinguishability operators on $X_{1}, \ldots, X_{n}$ respectively. Then, the two fuzzy relations $T\left(E_{1}, \ldots, E_{n}\right)$ and $\operatorname{Min}\left(E_{1}, \ldots, E_{n}\right)$ on $X_{1} \times \cdots \times$ $X_{n}$ defined for all $\left(x_{1}, \ldots, x_{n}\right),\left(y_{1}, \ldots, y_{n}\right) \in X_{1} \times \ldots \times X_{n}$ by

$$
\begin{gathered}
T\left(E_{1}, \ldots, E_{n}\right)\left(\left(x_{1}, \ldots, x_{n}\right),\left(y_{1}, \ldots, y_{n}\right)\right)= \\
T\left(E_{1}\left(x_{1}, y_{1}\right), \ldots, E_{n}\left(x_{n}, y_{n}\right)\right)
\end{gathered}
$$

and

$$
\begin{gathered}
\operatorname{Min}\left(E_{1}, \ldots, E_{n}\right)\left(\left(x_{1}, \ldots, x_{n}\right),\left(y_{1}, \ldots, y_{n}\right)\right)= \\
\operatorname{Min}\left(E_{1}\left(x_{1}, y_{1}\right), \ldots, E_{n}\left(x_{n}, y_{n}\right)\right)
\end{gathered}
$$

are $T$-indistinguishability operators on $X_{1} \times \ldots \times X_{n}$.

Proposition 3.10: Let $E$ be a $T$-indistinguishability on $[0,1]$ and $h$ an aggregation operator. $h$ is $E$-Lipschitzian if and only if $h_{(n)}$ (as a fuzzy subset of $[0,1]^{n}$ ) is extensional with respect to $T(\overbrace{E, \ldots, E}^{n \text { times }})$ for all $n \in \mathbb{N}$.

Proof: Proposition 2.14

Lemma 3.11: [2] Let $T$ be a continuous t-norm. The for all $x, y \in[0,1] x \geq y$

$$
T(x, \vec{T}(x \mid y))=y
$$

Next Proposition shows that a t-norm $T$ is an $E_{T^{-}}$ Lipschitzian aggregation operator.

Proposition 3.12: Let $T$ be a continuous t-norm. Then $T$ is an $E_{T}$-Lipschitzian aggregation operator.

Note that if $x_{i} \leq y_{i}$ for all $i=$ $1, \ldots n$, then $T\left(E_{T}\left(x_{1}, y_{1}\right), \ldots, E_{T}\left(x_{n}, y_{n}\right)\right)=$ $E_{T}\left(T\left(x_{1}, \ldots, x_{n}\right), T\left(y_{1}, \ldots, y_{n}\right)\right)$. Since for every tnorm different from the Minimum $E_{T}^{p}<E_{T}^{q}$ if $p>q$, we have that $T \neq M$ in is not $E_{T}^{p}$-Lipschitzian for $p<1$.

If $T$ is a continuous Archimedean t-norm, the $E_{T^{-}}^{p}$ Lipschitzian property becomes a classical Lipschitzian condition.

Proposition 3.13: Let $T$ be a continuous Archimedean t-norm with additive generator $t, p \in[0,1]$ and $h$ an aggregation operator. $h$ is $E_{T}^{p}$-Lipschitzian if and only if $\forall n \in \mathbb{N}, \forall x_{1}, \ldots, x_{n}, y_{1}, \ldots, y_{n} \in[0,1]$

$$
\begin{aligned}
& p\left|t\left(x_{1}\right)-t\left(y_{1}\right)\right|+\ldots+p\left|t\left(x_{n}\right)-t\left(y_{n}\right)\right| \geq \\
& \left|t\left(h\left(x_{1}, \ldots, x_{n}\right)\right)-t\left(h\left(y_{1}, \ldots, y_{n}\right)\right)\right|(1) .
\end{aligned}
$$

Proof:

$$
\begin{gathered}
t^{[-1]}\left(t\left(t^{-1}\left(p\left|t\left(x_{1}\right)-t\left(y_{1}\right)\right|\right)\right)+\ldots+t\left(t^{-1}\left(p\left|t\left(x_{n}\right)-t\left(y_{n}\right)\right|\right)\right)\right) \leq \\
t^{-1}\left(\left|t\left(h\left(x_{1}, \ldots, x_{n}\right)\right)-t\left(h\left(x_{1}, \ldots, x_{n}\right)\right)\right|\right) \\
t^{[-1]}\left(p\left|t\left(x_{1}\right)-t\left(y_{1}\right)\right|+\ldots+p\left|t\left(x_{n}\right)-t\left(y_{n}\right)\right|\right) \leq \\
t^{-1}\left(\left|t\left(h\left(x_{1}, \ldots, x_{n}\right)\right)-t\left(h\left(x_{1}, \ldots, x_{n}\right)\right)\right|\right)
\end{gathered}
$$

$$
\begin{aligned}
& p\left|t\left(x_{1}\right)-t\left(y_{1}\right)\right|+\ldots+p\left|t\left(x_{n}\right)-t\left(y_{n}\right)\right| \\
& \geq\left|t\left(h\left(x_{1}, \ldots, x_{n}\right)\right)-t\left(h\left(x_{1}, \ldots, x_{n}\right)\right)\right| .
\end{aligned}
$$

Last Proposition says that for all $n \in \mathbb{N}$, the map $H$ : $[0, t(0)]^{n} \rightarrow[0, t(0)]$ defined by

$$
H\left(x_{1}, \ldots, x_{n}\right)=t\left(h\left(t^{-1}\left(x_{1}\right), \ldots, t^{-1}\left(x_{n}\right)\right)\right)
$$

is a $p$-Lipschitzian map.

Also note that if $T$ is the Lukasiewicz t-norm, then (1) is the definition of the Lipschitz property in [13], so that Definition 3.8 contains the one in [13] as a particular case.

If an aggregation operator $h$ is $E_{T}^{p}$-Lipschitzian, it may happen that for different values of $n$ the corresponding $n$ ary operators $h_{(n)}$ may satisfy the Lipschitzian conditions for different values of $p$ ([4] p. 12).

Definition 3.14: An aggregation operator is subidempotent if and only if for all $x \in[0,1]$ and $n \in \mathbb{N}$, $n$ times $h(\overbrace{x, \ldots, x}) \leq x$

Proposition 3.15: Let $T \neq \operatorname{Min}$ be a t-norm, $h$ a subidempotent aggregation operator and $n \in \mathbb{N}$. If $h_{(n)}$ is $E_{T^{-}}^{p}$ Lipschitzian, then $p \geq \frac{1}{n}$.

Proof: If $h_{(n)}$ is $E_{T}^{p}$-Lipschitzian, then in particular, for $x \in X$

$T(\overbrace{E_{T}^{p}(1, x), \ldots, E_{T}^{p}(1, x)}^{n \text { times }} \leq E_{T}(h(\overbrace{1, \ldots, 1)}^{n \text { times }}, h(\overbrace{x, \ldots, x)}^{n \text { times }})$

and so

$$
x_{T}^{(p n)} \leq h \overbrace{(x, \ldots, x)}^{n \text { times }} \leq x
$$

which holds if and only if $p n \geq 1$ or equivalently, if and only if $p \geq \frac{1}{n}$

For $T$ is a strict continuous Archimedean t-norm the subidempotent property trivially holds.

Proposition 3.16: Let $T$ be a strict continuous Archimedean t-norm with additive generator $t, h$ an aggregation operator and $n \in \mathbb{N}$. If $h_{(n)}$ is $E_{T}^{p}$-Lipschitzian, then $p \geq \frac{1}{n}$.

Proof: Taking $x_{i}=1$ and $y_{i}=0$ for all $i=1, \ldots, n$ in Proposition 3.13, we get

$$
\begin{gathered}
p|t(1)-t(0)|+\ldots+p|t(1)-t(0)| \geq|t(1)-t(0)| . \\
n p t(0) \geq t(0)
\end{gathered}
$$

or

$$
p \geq \frac{1}{n}
$$


In [4], it has been proved that the arithmetic mean is the only aggregation operator $h$ whose $n$-ary maps $h_{(n)}$ are $\frac{1}{n}$-Lipschitzian. Proposition 3.22 generalizes this result to arbitrary quasi-arithmetic means.

Next Proposition is well known.

Proposition 3.17: [1], [18] $m$ is a quasi-arithmetic mean in $[0,1]$ if and only if there exists a continuous monotonic map $t:[0,1] \rightarrow[-\infty, \infty]$ such that for all $n \in \mathbb{N}$ and $x_{1}, \ldots, x_{n} \in[0,1]$

$$
m\left(x_{1}, \ldots, x_{n}\right)=t^{-1}\left(\frac{t\left(x_{1}\right)+\ldots+t\left(x_{n}\right)}{n}\right) .
$$

$m$ is continuous if and only if $\operatorname{Ran} t \neq[-\infty, \infty]$.

Lemma 3.18: [11] Let $t, t^{\prime}:[0,1] \rightarrow[-\infty, \infty]$ be two continuous strict monotonic maps with $\operatorname{Ran} t, \operatorname{Ran} t^{\prime} \neq$ $[-\infty, \infty]$ differing only by a non-zero multiplicative constant $\alpha\left(t^{\prime}=\alpha t\right)$ and $m_{t}, m_{t^{\prime}}$ the quasi-arithmetic means generated by them respectively. Then $m_{t}=m_{t^{\prime}}$.

Lemma 3.19: [11] Let $t, t^{\prime}:[0,1] \rightarrow[-\infty, \infty]$ be two continuous strict monotonic maps with $\operatorname{Ran} t, \operatorname{Ran} t^{\prime} \neq$ $[-\infty, \infty]$ differing only by an additive constant and $m_{t}, m_{t^{\prime}}$ the quasi-arithmetic means generated by them respectively. Then $m_{t}=m_{t^{\prime}}$.

Lemma 3.20: [11] Let $t:[0,1] \rightarrow[-\infty, \infty]$ be a continuous strict monotonic map. Then $m_{t}=m_{-t}$.

Proposition 3.21: [11] The map assigning to every continuous Archimedean t-norm $T$ with generator $t$ the mean $m_{t}$ generated by $t$ is a bijection between the set of continuous Archimedean t-norms and the set of continuous quasiarithmetic means.

Proposition 3.22: Let $T$ be a continuous Archimedean tnorm with additive generator $t$ and $m_{t}$ the quasi-arithmetic mean generated by $t$.

- (a) For every $n \in \mathbb{N} m_{t(n)}$ is $E_{T}^{p}$-Lipschitzian if and only if $p \geq \frac{1}{n}$.

- (b) $m_{t}$ is the only aggregation operator fulfilling (a)

In Proposition 3.12 we have proved that a t-norm $T$ is $E_{T}$-Lipschitzian. In fact, $T_{(n)}$ can also be seen as a fuzzy point of $[0,1]^{n}$ and a fuzzy map from $[0,1]^{n-1}$ into $[0,1]$.

Definition 3.23: Let $E$ be a $T$-indistinguishability operator on a set $X$, a fuzzy subset of $X \mu$ is a fuzzy point of $X$ with respect to $E$ if and only if for all $x, y \in X$

$$
T(\mu(x), \mu(y)) \leq E(x, y) .
$$

Proposition 3.24: Let $T$ be a continuous t-norm. $T_{(n)}$ is a fuzzy point of $[0,1]^{n}$ with respect to $T(\overbrace{\left.E_{T}, \ldots, E_{T}\right)}^{n \text { times }}$.

Proof: We have to prove that

$$
T\left(T\left(x_{1}, \ldots, x_{n}\right), T\left(y_{1}, \ldots, y_{n}\right)\right) \leq
$$

$$
T\left(E_{T}\left(x_{1}, y_{1}\right), \ldots, E_{T}\left(x_{n}, y_{n}\right)\right)
$$

which is an immediate consequence of

$$
T\left(x_{i}, y_{i}\right) \leq E_{T}\left(x_{i}, y_{i}\right) \text { for all } i=1, \ldots, n \text {. }
$$

Definition 3.25: Let $E, F$ be two $T$-indistinguishability operators on $X$ and $Y$ respectively and $R$ a fuzzy set of $X \times Y$ (i.e.: $R: X \times Y \rightarrow[0,1])$. $R$ is a fuzzy map from $X$ to $Y$ if and only if for all $x, x^{\prime} \in X, y, y^{\prime} \in Y$

- (a) $T\left(E\left(x, x^{\prime}\right), F\left(y, y^{\prime}\right), R(x, y)\right) \leq R\left(x^{\prime}, y^{\prime}\right)$

- (b) $T\left(R(x, y), R\left(x, y^{\prime}\right)\right) \leq F\left(y, y^{\prime}\right)$.

Proposition 3.26: Let $T$ be a continuous t-norm. $T_{(n)}$ is a fuzzy map from $[0,1]^{n-1}$ to $[0,1]$ endowed with the $T$ indistinguishability operators $T(\overbrace{\left.E_{T}, \ldots, E_{T}\right)}^{n-1 \text { times }}$ and $E_{T}$ respectively.

In fact, it can be proved in the same way that $T_{(n)}$ is a fuzzy map from $[0,1]^{k}$ to $[0,1]^{n-k}(2 \leq k \leq n-1)$ endowed with the $T$ indistinguishability operators $T(\overbrace{E_{T}, \ldots, E_{T}}^{k \text { times }})$ and $T(\overbrace{\left.E_{T}, \ldots, E_{T}\right)}^{n-k \text { times }}$ respectively.

Kernel aggregation operators are a family of aggregation operators tightly related to Lipschitzian ones. They were introduced in [17] (see also [13], [4]). As the Lipschitzian condition, the condition for being a kernel operator was related to the usual metric on the unit interval. It can be extended using natural indistinguishability operators in the same way as it has been done in this paper with the Lipschitzian condition. Again, if the $T$ norm is the Lukasiewicz one, the original definition of [17] is recovered.

Definition 3.27: Let $E$ be a $T$-indistinguishability operator on $[0,1]$ and $h$ an aggregation operator. $h$ is an $E$-kernel aggregation operator if and only if $\forall n \in \mathbb{N}$, $\forall x_{1}, \ldots, x_{n}, y_{1}, \ldots, y_{n} \in[0,1]$

$$
\begin{aligned}
& \operatorname{Min}\left(E\left(x_{1}, y_{1}\right), \ldots, E\left(x_{n}, y_{n}\right)\right) \\
\leq & E_{T}\left(h\left(x_{1}, \ldots, x_{n}\right), h\left(y_{1}, \ldots, y_{n}\right)\right) .
\end{aligned}
$$

Proposition 3.28: Let $E$ be a $T$-indistinguishability operator on $[0,1]$ and $h$ an aggregation operator. $h$ is an $E$-kernel aggregation operator if and only if $h_{(n)}$ (as a fuzzy subset of $\left.[0,1]^{n}\right)$ is extensional with respect to $\operatorname{Min}(\overbrace{E, \ldots, E}^{n \text { times }})$ for all $n \in \mathbb{N}$.

\section{Proof: Proposition 2.14}

For Archimedean t-norms, the kernel property can be written as follows.

Proposition 3.29: Let $T$ be a continuous Archimedean t-norm with additive generator $t, p \in[0,1]$ and $h$ an 
aggregation operator. $h$ is $E_{T}^{p}$-kernel aggregation operator if and only if $\forall n \in \mathbb{N}, \forall x_{1}, \ldots, x_{n}, y_{1}, \ldots, y_{n} \in[0,1]$

$$
\begin{aligned}
& \operatorname{Max}\left(p\left|t\left(x_{1}\right)-t\left(y_{1}\right)\right|, \ldots, p\left|t\left(x_{n}\right)-t\left(y_{n}\right)\right|\right) \\
\geq & \left|t\left(h\left(x_{1}, \ldots, x_{n}\right)\right)-t\left(h\left(y_{1}, \ldots, y_{n}\right)\right)\right|
\end{aligned}
$$

Proof:

$$
\begin{gathered}
\operatorname{Min}\left(t^{-1}\left(p\left|t\left(x_{1}\right)-t\left(y_{1}\right)\right|\right), \ldots, t^{-1}\left(p\left|t\left(x_{n}\right)-t\left(y_{n}\right)\right|\right)\right) \leq \\
t^{-1}\left(\left|t\left(h\left(x_{1}, \ldots, x_{n}\right)\right)-t\left(h\left(x_{1}, \ldots, x_{n}\right)\right)\right|\right) \\
t^{-1}\left(\operatorname{Max}\left(p\left|t\left(x_{1}\right)-t\left(y_{1}\right)\right|, \ldots, p\left|t\left(x_{n}\right)-t\left(y_{n}\right)\right|\right)\right) \leq \\
t^{-1}\left(\left|t\left(h\left(x_{1}, \ldots, x_{n}\right)\right)-t\left(h\left(x_{1}, \ldots, x_{n}\right)\right)\right|\right) \\
\operatorname{Max}\left(p\left|t\left(x_{1}\right)-t\left(y_{1}\right)\right|, \ldots, p\left|t\left(x_{n}\right)-t\left(y_{n}\right)\right|\right) \geq \\
\left|t\left(h\left(x_{1}, \ldots, x_{n}\right)\right)-t\left(h\left(x_{1}, \ldots, x_{n}\right)\right)\right| .
\end{gathered}
$$

If $T$ is the Lukasiewicz t-norm and $p=1$, then (4) is the definition of the kernel aggregation operator introduced in [17].

\section{CONCLUSIONS}

In this paper Lipschitzian and kernel aggregation operators with respect to the natural $T$-indistinguishability operator $E_{T}$ and their powers have been studied.

It has been proved that a t-norm $T$ is $E_{T}$-Lipschitzian, and it is also a fuzzy point and a fuzzy map as well

Quasi-arithmetic means $m_{t}$ play an important role since they are the more stable aggregation operator with respect to $T$, meaning that the corresponding $n$-ary operators $m_{t(n)}$ are $E_{T}^{\frac{1}{n}}$-Lipschitzian maps.

Lipschitzian and kernel properties are not only interesting for aggregation operators, but also in most of th areas where fuzzy reasoning is present. Therefore, they deserve a deeper study.

\section{ACKNOWLEDGMENT}

This work was partially supported in part by DGYCYT project number TIN2006-14311.

\section{REFERENCES}

[1] J. Aczel (1966) Lectures on functional equations and their applications. Academic Press. New York/London.

[2] D. Boixader (1998) Some Properties Concerning the Quasi-inverse of a t-norm. Mathware and Soft Computing, vol. 5, pp. 5-12.

[3] D. Boixader, J. Jacas, J. Recasens (2000). Fuzzy Equivalence Relations: Advanced Material. In Dubois, Prade Eds. Fundamentals of Fuzzy Sets, Kluwer, pp. 261-290.

[4] T. Calvo, A. Kolesárova, M. Komorníková, R. Mesiar (2002). Aggregation Operators: Properties, Classes and Construction Methods. In Mesiar, Calvo, Mayor Eds. Aggregation Operators: New Trends and Applications. Studies in Fuzziness and Soft Computing. Springer, pp. 3-104.

[5] T. Calvo, R. Mesiar (2001). Stability of aggregation operators. Proc EUSFLAT 2001, Leicester. pp. 457-458.

[6] M. Demirci (2002). Fundamentals of M-vague algebra and M-vague arithmetic operations International Journal of Uncertainty, Fuzziness and Knowledge-Based Systems, vol. 10, pp. 25-75.

[7] P. Hájek (1998) Metamathematics of Fuzzy Logic. Kluwer. Dordrecht.
[8] J. Jacas (1993). Fuzzy topologies induced by S-metrics. The Journal of Fuzzy Mathematics vol. 1, pp. 173-191.

[9] J. Jacas, J. Recasens (2000). One dimensional indistinguishability operators. Fuzzy Sets and Systems, vol. 109, pp. 447-451.

[10] J. Jacas, J. Recasens (2003) Aggregation of T-Transitive Relations. Int J. of Intelligent Systems, vol. 18, pp. 1193-1214.

[11] J. Jacas, J. Recasens (2006) Aggregation Operators Based on Indistinguishability Operators. Int J. of Intelligent Systems, vol. 21, pp. 857873.

[12] J. Jacas, J. Recasens (2006)Linguistic Modifiers, Fuzzy Maps and the lipschitzian Condition. Proc IPMU 2006, Paris.

[13] A. Kolesárová, J. Mordelová (2001) 1-Lipschitz and kernel aggregation operators. Proc AGOP 2001, Oviedo pp. 71-76.

[14] A. Kolesárová, J. Mordelová, E.Muel (2004). Kernel aggregation. operators and their marginals. Fuzzy Sets and Systems, vol. 142, pp. 35 50.

[15] C. M. Ling (1965) Representation of associative functions Publ. Math. Debrecen, vol. 12, pp. 189-212.

[16] V, Novák, R. Mesiar (1999). Operations fitting triangular-norm-based biresiduation. Fuzzy Sets and Systems, vol. 104, pp. 77-84.

[17] J. Mordelová, E. Muel (2001) Kernel aggregation operators. Proc AGOP 2001, Oviedo pp. 95-98.

[18] E. P. Klement, R. Mesiar, E. Pap (2000). Triangular norms. Kluwer. Dordrecht.

[19] B. Schweizer, A. Sklar (1983) Probabilistic Metric Spaces. NorthHolland. Amsterdam.

[20] A.R. De Soto, J. Recasens (2000). Modeling a Linguistic Variable as a Hierarchical Family of Partitions induced by an Indistinguishability Operator. Fuzzy Sets and Systems, vol. 121, pp. 427-437.

[21] L.Valverde (1985). On the structure of F-indistinguishability operators, Fuzzy Sets and Systems, vol. 17, pp. 313-328.

[22] L.A.Zadeh (1971). Similarity relations and fuzzy orderings, Information Science, vol. 3, pp. 177-200. 